\title{
SOME NEGATIVE EFFECTS OF WARNINGS
}

\author{
S. David Leonard \\ University of Georgia \\ Athens, GA, USA \\ E-mail: dleonard@uga.edu
}

\begin{abstract}
Summary: Warnings provide important information about hazards that may be encountered by individuals exposed to them. Some warnings may not be effective because they are not interpreted correctly, require people to perform unrealistic activities, are not recognized, or are ignored because they have lost their attentiongetting capabilities. A common occurrence in the USA, and perhaps other countries as well, is the use of signs to warn of work being performed on the roadway ahead. Often such signs are not removed after the work has been done. Consequently, drivers who have had the experience of seeing the signs with no workers may come to ignore them, and when there is a work crew on the road, some accidents may occur. In the context of modern learning theory, the extinction of cautionary behavior would be expected under such circumstances. The present study surveyed the behaviors of 224 respondents, many of whom indicated they tended to ignore such signs. The present study examined behavior reported to occur under various conditions and found evidence that the greater the frequency of unfulfilled expectations, the greater the reported likelihood of ignoring the warnings.
\end{abstract}

\section{INTRODUCTION}

Warnings are an important and effective means of helping people avoid hazards in a variety of situations. However, care must be exercised in the use of warnings. As noted by Leonard and Karnes (2005) warnings cannot overcome strongly engrained behavioral patterns or inept designs. Further, care must be taken to avoid overwhelming the individual with too many warnings at once (cf. Frantz, Rhoades, Young \& Schiller, 1999). In general, warnings may describe behaviors necessary to avoid the hazards they are describing. However, in some situations it may be assumed that the observers of the warning can adapt their behaviors to avoid possible negative consequences from the hazards, e.g., a sign saying "Bridge out" should not require the statement "use brake" for individuals licensed to drive. Obedience to the procedures described in the warning may occur in the absence of previous experience with the hazardous product itself, because the term warning connotes the need for exercising care or because one's experience in other situations generalizes to the present. For example, a warning about a paint remover might state, "WARNING: highly flammable. Do not use near open flame!" A person working with the substance may heed the warning, even though he has never experienced the sight of flames involving the substance, because he might be aware of fires associated with other flammable substances such as gasoline. Of course, one may fail to heed the warning, because previous experience with similar materials did not produce the effect warned against. Further, in other circumstances, warnings may have considerably less effect, because individuals having been warned assume the warning is more concerned with the convenience of someone avoiding dealing with intrusions while at work. In some cases this assumption may be correct. Leonard 
and Karnes (1998) found several factors that affected people's tendency to follow or not to follow some warnings. Obviously, some of these behaviors are related to specific situations, but there is the possibility that they may carry over to other similar situations inappropriately. In examining the situations that occur on the roadways where warnings of workmen ahead are commonly posted, it has been noted that often there is no work being done and there are no workers. This might lead one to conclude that while no workers are ahead, the signs are left in place for the convenience of the workers, should they choose to be there at some time convenient for them. This paper is concerned with the effects of various events that may cause warnings to lose their effectiveness as a result of the extinction of the cautionary response in the observer.

Aesop's fable about the little boy who cried "wolf" when there was no wolf and, therefore, did not get help when the wolf actually showed up is well known. Although the wisdom of the fable has been accepted throughout the ages, and procedures to encourage or discourage certain behaviors were used by animal trainers and others over that time, only at the end of the nineteenth century has scientific documentation of the principles of conditioning and extinction been produced (cf. Pavlov, 1927; Thorndike, 1898). Since that time hundreds of scientific papers have been published describing various factors involved in conditioning phenomena in a variety of species including humans (cf. Chance, 1994). However, there are still many everyday situations in which the existence of conditioning principles is ignored. For example, parents may punish incorrect behavior in an inconsistent manner, thus failing to extinguish the tendency for the incorrect behavior. The need for modification of behavior on the roads is relevant in many ways. As noted by Hancock and de Ridder (2003) "...the vast majority of safety resources which to date have been directed to the accident question have focused overwhelmingly on crash survival," but the importance of avoiding the accident is perhaps more important. Thus, warnings that encourage drivers to behave in ways that can avoid accidents are important.

The present study deals with the unintended consequences of providing warnings in circumstances where there are no hazards or where hazards are temporary but the warnings do not reflect that. Specifically, the study concerns highway warnings that are not associated with the presence of hazards of the type described. The most serious of these problems is the use of "road work" or "men at work" signs. Some of these are often left in place not only over brief periods such as weekends but for weeks or months after the work is finished. Interestingly, many more drivers than workers are injured or killed at actual work sites. This likely results from the failure to avoid heavy equipment or rollovers and running off of the road in a last moment attempt to avoid some sort of collision.

Another situation in which information presented seems to be at variance with the existing circumstances is the use of speed warnings on signs indicating curves. In many instances the speed indicated is far lower than would be a safe speed to negotiate the curve. Therefore, drivers may be less likely to lower speeds when the sign's value is veridical. This study involved a survey of how people performed in conjunction with the road work signs and signs indicating the appropriate speeds for curves. 


\section{METHOD}

\section{Participants}

Participants were students at the University of Georgia (UGA). Of the 224 participants, 112 were men and 112 were women. Their ages ranged from 17 to 27 with a median of 19 . They participated in groups ranging in size from 3 to 10 persons, and they received credit for participation.

\section{Table 1. Questions Regarding the Reactions to Occurrence of Road Work Signs}

1. When driving on the highway have you encountered signs indicating that work was being done on the road ahead only to see no sign of workers?

Frequently Often Occasionally Seldom Never

2. Suppose you see signs indicating road work ahead. You would likely conclude there is no work occurring after driving as little as

More than $5 \mathrm{Mi} \quad 4-5 \mathrm{Mi}$ 3-4 Mi _ 2-3 Mi $1-2 \mathrm{Mi}$ 1 Mi or less

3. When driving in an unfamiliar area and you see a sign that road work is occurring, do you

a. Slow down immediately upon seeing the sign that says "ROAD WORK AHEAD"

b. Not slow down, but drive more cautiously.

c. Finally slow down only when you get to workers in the area.

d. Slow down only when a flagman shows a SLOW sign.

4. Suppose you have driven over an area for a number of times and have seen signs saying road work ahead. If you haven't seen any work being done during those times, do you

a. Slow down immediately upon seeing the sign that says "ROAD WORK AHEAD"

b. Not slow down, but drive more cautiously.

c. Finally slow down only when you get to workers in the area.

d. Slow down only when a flagman shows a SLOW sign.

5. Suppose you have driven over an area for a number of times and have seen signs saying road work ahead, but you haven't seen any work being done. If you are traveling through that area at night are you

a. More concerned about the possible hazards of road work than during the day.

b. Less concerned about the possible hazards of road work than during the day.

c. Neither $\boldsymbol{a}$ nor $\boldsymbol{b}$.

6. If you had never experienced signs advertising road work without having the road work appear, would you have started to slow down earlier upon seeing the signs? $\mathbf{Y} \mathbf{N}$

7. How do you respond to the limits attached to signs indicating a curve?

a. Follow them rigorously.

b. Let your view of the curve dictate the response.

c. Attend to them only if the limit is very low.

d. Usually ignore them.

8. Does the presence of speed limit signs on the same post as the curve symbol make you think many of them are irrelevant? $\mathbf{Y} \mathbf{N}$ 


\section{Materials and procedure}

The participants were asked to complete a survey on automotive behavior that included questions on demographic data, as well as questions relating to their behavior in various automotive situations. For this study the relevant items regarding driving behavior were as displayed in Table 1. The numbers on the items do not correspond to their positions in the survey but are provided for simplification of exposition. Some participants failed to answer some items; however, these seemed to be slips rather than attempts to avoid the questions, so all remaining responses were included in the analyses. Note that Item 1 requests an estimate of how often the event of no workers occurs when the signs are seen. One basis for use of word references rather than a percentage reference is that people who become attuned to the problem may be more likely to notice occurrences more readily than others. Thus the same frequencies may elicit different responses for different individuals based on the relevance to the particular individual.

\section{RESULTS AND DISCUSSION}

The data for males and females were quite similar. Although on average, males tended to assume the signs were not valid after a somewhat shorter distance than women, the difference was not statistically dependable. Women tended to be more likely to assume that the occurrence of the invalid signs affected their likelihood of slowing early, but the difference was not statistically reliable. No other data suggested sex differences. Therefore, the analyses described here combined data from both sexes.

Some of the responses to questions 1 through 8 were also examined in terms of some demographic data. Respondents were asked how often they drove on the highways each week. It could be assumed that frequency of highway travel might influence the perception of frequency of seeing road work signs that did not involve work being done. However, an analysis examining the relationship of high frequency travelers (more than two times per week, $n=75$ ) versus those who traveled infrequently $(2$ or fewer times per week, $n=127)$ did not find a dependable relationship, $\chi_{2}{ }^{2}=0.90, p>.25$. Perceived frequency of occurrence of signs without workers might also produce earlier assumptions of that no work exists when signs are seen. There were 223 responses to Item 2, the question of whether or not the participants had encountered road work signs without seeing any work occurring. Of these, 74 respondents checked "frequently," 96 checked "often," 50 checked "occasionally," and 3 checked "seldom." No participants indicated they had never observed such a situation. To determine whether or not their perceptions of frequency of occurrence affected their estimates of how far they would continue driving before concluding there was no work being done, the data for distances driven were divided into two groups: responses of over three miles (82) and three miles or less (138). The numbers of responses in those groups as a function of the perceptions of frequency are shown in Table 2. The relationship between distance driven and perception of frequency did not show a dependable effect, $\chi_{2}{ }^{2}=2.62, p>.10$. 
Table 2. Distance Traveled before Assuming Signs Were Not Valid as a Function of the Perceived Frequency of Such Signs

\begin{tabular}{lcccc}
\hline & & \multicolumn{3}{c}{ Perceived Frequency of Invalid Signs } \\
Distance Traveled & Frequently & Often & Occasionally & Seldom \\
Zero to three Miles & 41 & 63 & 34 & 2 \\
Over three miles & 33 & 33 & 16 & 1 \\
\hline
\end{tabular}

As may be seen in Table 3, responses to Items 3 and 4 indicated very few respondents slowed down immediately upon seeing the signs saying workers were ahead. However, the responses were rather different to an unfamiliar situation versus one in which they had previous experience, $\chi_{2}{ }^{2}=60.90, p<.001$. This result supports the argument that there is extinction of the tendency to be cautious when the invalid signs are often observed. The fact that only 25 percent of the respondents indicated they slowed down immediately when seeing the road work signs in unfamiliar circumstances supports the notion that this tendency generalizes across situations.

\section{Table 3. Comparison of Response Percentages Regarding Familiar versus Unfamiliar Areas}

\begin{tabular}{lcccc}
\hline & & \multicolumn{3}{c}{ Type of slowing response } \\
& Immediate & More cautious & See workers & Flagman shows \\
SLOW & & & & \\
Unfamiliar area & 25 & 54 & 42 & 1 \\
Familiar area & 5 & 45 & 42 & 8 \\
\hline
\end{tabular}

In response to the question of whether or not one would slow down earlier if one had never seen the signs without the workers present, the "yes" replies were overwhelming. There were 175 "yeses" and only 35 "no" responses, $z=9.66, p<.001$. A few participants failed to answer this item, perhaps because the format was slightly different from the preceding items; however, even had the non-responders all replied "no," the difference would have been highly dependable. A test of whether or not the perception of frequency of occurrence of invalid signs might have affected these responses did not indicate a dependable effect, $\chi_{2}{ }^{2}=2.86, p>.10$.

Responses to the questions about speed indicators on curve signs were somewhat similar in nature to the responses to the road work signs. Although relatively few respondents indicated they thought those signs were irrelevant ( 73 versus 149, a dependable difference, $z=5.10, p<$ .001 ), the responses to Item 7 concerning how one responded to curves mirrored the responses about slowing when seeing the road work signs. Only 19 percent of respondents indicated they followed the limits rigorously, while 69 percent said their responses were based on their view of the curve. Some respondents ( 8 percent) indicated they attended to the speed information only if it was very low, and 4 percent said they usually ignored the signs. This suggests that these signs are not considered reliable indicators of the actual safety requirements. The danger, of course, is that one might not consider a speed indication to be accurate and fail to make the appropriate adjustment, thus, causing an accident. 


\section{CONCLUSIONS}

The responses of these individuals indicate that the presence of warnings that are not accompanied by the hazardous situations that would be expected can dilute the influence of the warnings. Unfortunately, the effects of such dilution of impact may reduce the effectiveness of the warnings in places other than where the relative extinction is generated. Indeed, the accidents that might occur could affect workers who have been scrupulous in removing signs that are no longer valid. Obviously, an accident because of failure to heed the signs will occur at a location in which there is work going on. Thus, it is important to have some uniformity in the use of such signs. In most cases it is possible to use temporary portable signs that can be in place only when needed and then removed. While long-term projects may be the occasion for some semipermanent warnings, two factors must be considered: how long is the project to continue and how frequently is work being done. The author observed a set of signs on several days a week for several months without seeing any work occurring. It is possible to ameliorate the problem in several ways and by a variety of procedures. If work is only being done on weekends, that could be noted on the signs. One possibility to avoid showing signs when work is not being done is to cover the warnings when they are not relevant. Another is to use signs such as those relevant for certain seasons that are hinged so that the top half can be lowered to cover the bottom when not in use. In some cases light signs that can be turned on only when appropriate could be used. Such procedures are low in cost, but might provide high dividends in terms of enhancing driver attention to the messages and, consequently, reduction of accidents involving injuries and possibly deaths.

It is possible that the effects obtained in this study understate the overall influence on the driving public, because the respondents were mostly young and less experienced with the presence of invalid signs than an older group. Thus, there might have been less extinction of the need for caution among these respondents than could have occurred in more experienced drivers. It is also possible that with experience drivers may be able to use other cues to evaluate the likelihood that there are workers ahead. More research in this area is necessary.

The data and analyses presented here suggest that regulations for the use of road work signs should be enacted on a universal level. Inasmuch as much automobile travel occurs across state lines, failure to be consistent is a recipe for disastrous results.

\section{ACKNOWLEDGMENTS}

The author wishes to thank Professors B. Edward Mulligan and Richard E. Burke for helpful comments and assistance in the preparation of the final manuscript.

\section{REFERENCES}

Chance, P. (1994). Learning and behavior. Pacific Grove, CA: Brooks/Cole.

Frantz, J.P., Rhoades, T.P., Young, S.P. and Schiller, J.A. (1999). Potential problems associated with overusing warnings. In Proceedings of the Human Factors and Ergonomics Society 43rd Annual Meeting-1999, 916-920.

Hancock, P.A., \& de Ridder, S.N. (2003). Behavioural accident avoidance science: 
Understanding response in collision incipient conditions. Ergonomics, 46(12), 1111-1135.

Leonard, S.D., \& Karnes, E.W. (1998). Influence of context on warnings. Advances in occupational ergonomics and safety 2. S. Kumar (Ed.). Amsterdam: IOS Press 104-107.

Leonard, S.D., \& Karnes, E.W. (2005). Why some warnings don't work. Proceedings if XIX Annual International Occupational Ergonomics and Safety Conference, 15-20.

Pavlov, I. (1927). Conditioned reflexes. Oxford: Oxford University Press.

Thorndike, E.L. (1898). Animal intelligence: An experimental study of the association processes of animals. Psychological Monographs No. 8. 